\title{
Linear Psoriasis-A rare presentation
}

\author{
M M Rahaman' , M A Islam², M R Ullah³ , M Z P Barbhuiyan"
}

1 Dr. Md. Mahabubur Rahaman DDV, FCPS (Dermatology \& VD) Cosmetic \& Hair transplant surgeon Aurora skin \& Aesthetics Dhanmondi, Dhaka.

2

2 Dr. Md. Aminul Islam MCPS ( Dermatology \& VD), FCPS (Dermatology \& VD) Assistant Professor Northern International Medical College

3 Dr. Md. Rahmat Ullah MCPS, DDV, FCPS (Dermatology \& VD) Dept. of Dermatology BSMMU

${ }^{4}$ Dr. Md. Zahed Parvez Barbhuiyan DDV, MPH

Cosmetic \& Hair transplant surgeon Consultant, ShSMCH

\section{Correspondence}

Dr. Md. Mahabubur Rahaman DDV, FCPS (Dermatology \& VD) Cosmetic \& Hair transplant surgeon Aurora skin \& Aesthetics Dhanmondi, Dhaka.

\section{Introduction}

Psoriasis is a common, chronic, inflammatory and proliferative skin disorder, affecting about $2 \%$ of the population. Linear psoriasis is an unusual subtype characterized by a distribution of the psoriatic lesions along Blaschko lines ${ }^{1}$. This rare form of psoriasis was first described in 1951. There are few cases reported in the literature $\mathrm{e}^{1,2,3}$ and therefore there is no estimated prevalence. Also because of its clinical and histological similarity to Linear Verrucous Epidermal Nevus (ILVEN), some cases are probably not reported, contributing to the under diagnosis. Pathogenesis of linear Psoriasis is not clear but it could be explained by the concept of genetic mosaicism. $^{2}$

Happle proposed the pathogenesis of linear psoriasis as somatic recombination of a gene predisposing to psoriasis leading to segmental mosaicism. This hypothesis offers a reasonable explanation for linear psoriasis being a nonhereditary trait and the linear distribution similar to many other mosaic skins disorders. ${ }^{3}$

\section{Case Report}

A 25 year-old male presented with a linear erythematous plaque on left upper extremity which extending from tip of the index to mid forearm for 2 years. There was no $\mathrm{H} / \mathrm{O}$ any episodes of infection preceding the dermatosis. He had no personal or family history of psoriasis.

Physical examination revealed a linear erythematous plaque covered with silvery scales. Auspitz sign (forcible removal of scale pinpoint bleeding occurs) was positive. Nail pitting on both finger \& toe nails were present. (Fig. $01 \& 02$ )

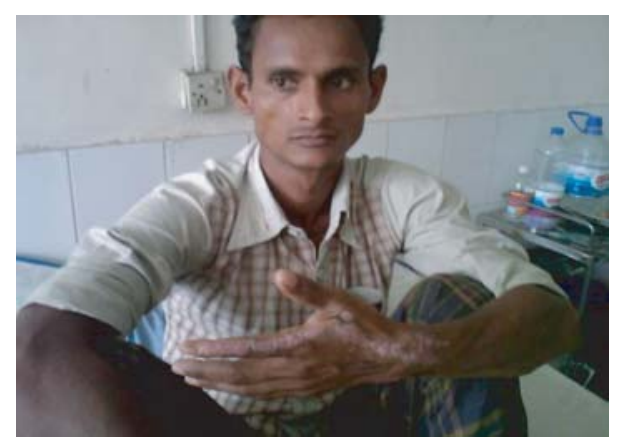

Figure 01: Photograph of linear lesion on left upper extremity extending from tip of the index to mid forearm

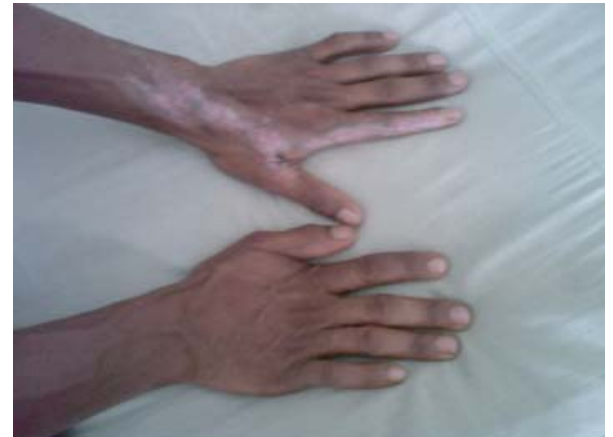

Figure-2: Close view

The histopathologic analysis of a skin biopsy revealed orthokeratosis, parakeratosis and spongiform pustules- Munro's micro abscesses, in association with a regular elongation of rete ridges, suprapapillary thinning, and an absence of granular cell layers (fig.3).

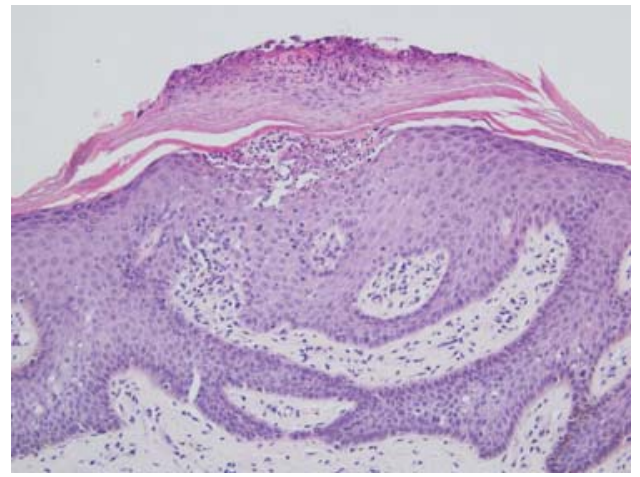

Figure-03 : Histopathological findings of lesional biopsy

The patient was treated with only potent topical steroid for one month. After the end of this period there was more than eighty percent skin lesion subsided but nail pitting persists.

\section{Discussion}

Linear psoriasis (LPS) is an exceedingly rare variant of psoriasis. LPs may be associated with non segmental plaques of psoriasis vulgaris (superimposed linear psoriasis type) or it can occur as an isolated lesion epidermal psoriasi form hyperplasia, superficial pallor with diminished granular layer, incipient subcorneal spongiform pustule formation and staggered 
collections of neutrophils in a thickened parakeratotic horn (linear psoriasis of the isolated type $)^{4}$.

Linear psoriasis may be confused with inflammatory linear verrucous epidermal nevus (ILVEN) or Koebners's response of psoriasis over verrucous epidermal nevus. ${ }^{5}$ Saraswat et al described the clinical differences between the two entities. ILVEN tends to develop during the first month of life, progresses slowly, can be very pruritic and is usually unresponsive to treatment. However, linear psoriasis tends to develop later in life, progresses rapidly, only occasionally pruritic and involvement of scalp and nails responds well to antipsoriatic treatment ${ }^{6}$. As for the histological features, ILVEN classically demonstrates hypergranulosis and parakeratosis alternating with hypogranulosis and orthokeratosis. ${ }^{7}$ On the other hand, the classic features of psoriasis are hyperkeratosis, parakeratosis, the absence of granular cell layers, an elongation of rete ridges, suprapapillary thinning and Munro's microabscesses, which can also be seen in ILVEN. Immunohistopathologic studies may be helpful in distinguishing the two conditions, as done by Vissers et al. $^{8}$ In ILVEN patients, the number of Ki-67-positive nuclei tended to be reduced, whereas the number of keratin-10-positive cells was increased compared with psoriasis patients. Involucrin is a marker of epidermal differentiation, a precursor protein of the cornfield envelope, and staining is used as a tool to distinguish between psoriasis and ILVEN is absent in ILVEN patients but detectable in psoriasis ${ }^{9}$

Systemic treatments for linear psoriasis are not recommended unless the skin lesions are particularly burdensome and cannot be controlled with topical agents or narrow-band ultraviolet B phototherapy. ${ }^{10}$

\section{Conclusion}

Our patient had onset of lesions in adult life and did not develop lesions of psoriasis elsewhere in the body and the lesions progressed only in a linear distribution. Histopathology revealed features of psoriasis and the lesions responded well to treatment.

\section{References}

1. Linear psoriasis; Wei Li, MD, Xiao-Yong Man, MD PhD:CMAJ April 17, 2012 vol. 184 no. 7 First published February 6, 2012, doi:10.1503/cmaj.110866.

2. Happle R. Linear psoriasis and ILVEN: Is lumping or splitting appropriate? Dermatology 2006;212:101-2. Cross Ref Medline Google Scholar.

3. Happle R. Somatic recombination may explain linear psoriasis. J Med Genet 1991;28(5):337.

4. Marc W. Levin MD Lehigh Valley Health Network, Marc_W.Levin@lvhn.org

5. Happle R. Linear psoriasis and ILVEN: is lumping or splitting appropriate? Dermatology2006;212(2):101-2.

6. Saraswat $A$, et al. Unilateral linear psoriasis with palmoplantar, nail, and scalp involvement. Pediatr Dermatol 2004; 21: 70 [PubMed].

7. Nidhi Singh, Naseem Noorunnisa; Linear psoriasis: A rare presentation: Indian Dermatology Online Journal 2012: 3 ( 1) : 71-73DOI: 10.4103/2229-5178.93489 PMID: 23130273.

8. Vissers WHPM, Muy L, van Erp PEJ, de Jong EMGJ, van de Kerkhof PCM. Immunohistochemical differentiation between inflammatory linear verrucous epidermal nevus (ILVEN) and psoriasis. Eur J Dermatol 2004;14:216-220.

9. Ana Brinca,* F. Santiago, D. Serra, P. Andrade, R. Vieira, and A. Figueiredo ;Linear Psoriasis - A Case Report; Case Rep Dermatol. 2011 Jan-Apr; 3(1): 8-12.Published online Jan 22, 2011. doi: 10.1159/000324190.

10. Menter A, Korman NJ, Elmets CA, et al. Guidelines of care for the treatment of psoriasis and psoriatic arthritis: case-based presentations and evidence-based conclusions. J Am Acad Dermatol 2011;65:137-74. Cross Ref Medline Google Scholar. 